\title{
HIPERPARATIROIDISMO PRIMARIO. PARATIROIDECTOMIA MINIINVASIVA VIDEOASISTIDA
}

\author{
PRIMARY HYPERTHYROIDISM. MINIMALLY INVASIVE VIDEO-ASSISTED PARATHYROIDECTOMY
}

\author{
Luis Gramática $(h)^{1,2}$, César E Palas Zúñiga ${ }^{3}$, Rodolfo Cecenarro ${ }^{4}$, Facundo Antueno ${ }^{4}$, Noelia Villablanca ${ }^{4}$
}

\section{Resumen:}

Objetivo. Mostrar nuestra experiencia realizando la paratiroidectomía miniinvasiva video-asistida con abordaje central para el tratamiento de pacientes con hiperparatiroidismo primario, sin utilizar el monitoreo intraoperatorio de paratohormona (mipth), basados únicamente en la concordancia de dos estudios de localización preoperatorios.

Material y métodos. Se seleccionaron 27 pacientes con diagnóstico de hiperparatiroidismo primario, a los cuales se les realizó previo su consentimiento para la realización de un abordaje miniinvasivo, una ecografía cervical y una centellografía sesta-MIBI como estudios de localización pre-operatorios, los cuales fueron concordantes en todo ellos. Ninguno presentó antecedentes familiares que hicieran sospechar una neoplasia endócrina múltiple (NEM), enfermedad tiroidea conocida, cervicotomía previa, ni sospecha de carcinoma. La cirugía se realizó a través de una incisión cervical central de $1,5 \mathrm{~cm}$. con el empleo del bisturí harmónico. El mipth no se realizó en ninguno de los casos.

Resultados. En todos los casos se confirmó la ubicación topográfica del adenoma. No registramos persistencias del hiperparatiroidismo ni recidivas después de un período de seguimiento de 42 meses. No hubo lesiones recurrenciales. El tiempo quirúrgico promedio fue de 24,5 minutos y todos los pacientes se fueron de alta antes de las 24 horas. El requerimiento analgésico fue mínimo, y el resultado estético satisfactorio.

Conclusión: La exploración unilateral miniinvasiva de las paratiroides con magnificación videoasistida sin mipth, bajo la guía de dos estudios de localización concordantes, es un procedimiento seguro y factible, con resultados comparables a los de la técnica estándar y con ventajas en lo estético, menor dolor postoperatorio y hospitalización reducida.

Palabras clave: paratiroidectomía; PTMIV; MIBI; hiperparatiroidismo primario; ecografía.

\section{Abstract:}

Objective. Show our experience performing the minimally invasive video-assisted parathyroidectomy with central approach for the treatment of patients with primary hyperparathyroidism without using intraoperative parathyroid hormone monitoring (IPTHM) based solely on the concordance of two preoperative localization studies.

Material and methods. An informed consent for the accomplishment of a minimally invasive approach was performed on 27 selected patients diagnosed with primary hyperparathyroidism who underwent preoperative cervical ultrasound and sesta-MIBI scintigraphy studies, which were consistent across them. None had a family history that might indicate a multiple endocrine neoplasia (MEN), known thyroid disease, previous neck incision, or suspicion of carcinoma. Surgery was performed through a $1.5 \mathrm{~cm}$ central cervical incision and the use of harmonic scalpel. IPTHM was not performed in either case.

Results. In all cases the topographic location of the adenoma was confirmed. No signs of hyperparathyroidism persistence or recurrence were registered after a follow-up period of 42 months. There were no recurrent nerve lesions. The average operative time was 24.5 minutes and all patients were discharged within 24 hours. The analgesic requirement was minimal and the cosmetic result satisfactory. Conclusion: The minimally invasive unilateral exploration of the parathyroids with video-assisted magnification without IPTHM, under the guidance of two concordant localization studies is safe and feasible, with comparable results to those of standard technique as well as aesthetic advantages, less postoperative pain and reduced hospitalization.

Keywords: parathyroidectomy; MIVAP; MIBI; primary hyperparathyroidism; ultrasound.

\footnotetext{
${ }_{2}^{1}$ Profesor Titular y Jefe de servicio

2 Email de contacto: Igramatica@yahoo.com

${ }^{3}$ Cirujano especialista

${ }^{4}$ Residente en Cirugía General
} 


\section{Introducción}

A principios de los 80 , basados en que el $85 \%$ de los hiperparatiroidismos primarios están causados por un adenoma único, Tiblin y cols. ${ }^{1}$ proponen el abordaje unilateral de esta patología, observando una reducción en el número de complicaciones y del tiempo operatorio. No obstante, no es hasta la década del 90 que Gagner $^{2}$ realiza la primera paratiroidectomía mini-invasiva por vía endoscópica, iniciando el camino de los abordajes mínimos y localizados de las glándulas paratiroides. En la última década, el perfeccionamiento de las imágenes de localización preoperatoria, de las técnicas de laboratorio, y de nuevas tecnologías han motivado a muchos cirujanos a perfeccionar las técnicas mini invasivas, demostrando que el HPT $1^{\circ}$ es una enfermedad ideal para este tipo de cirugía, ya que el tumor es casi siempre único, benigno y de pequeño tamaño ${ }^{3-4-5}$.

\section{Objetivo}

Mostrar nuestros resultados con la paratiroidectomía mini-invasiva videoasistida (PTMIV) en el tratamiento del hiperparatiroidismo primario en pacientes seleccionados, a través de un abordaje central único y sin monitoreo intraoperatorio de paratohormona (mipth).

\section{Material y métodos}

Entre Junio del 2005 y Diciembre del 2009, 27 pacientes, 25 mujeres y 2 hombres, con una edad promedio de $52 \pm 13$ años, diagnosticados de HPT $1^{\circ}$ fueron operados mediante una PTMIV unilateral con acceso central, por un único cirujano endócrino de experiencia. El diagnóstico HPT $1^{\circ}$ fue realizado en 20 pacientes mediante screenings de calcemia y en el resto por la presencia de manifestaciones clínicas, confirmándose por valores elevados de paratohormona (PTH) intacta. La calcemia preoperatoria promedio fue de $11.58 \pm 1,25 \mathrm{mg} / \mathrm{dl}$ y la PTH de $260,30 \pm 233,8 \mathrm{pg} / \mathrm{ml}$.

Como estudios de localización preoperatorios se realizó una ecografía cervical y un centellograma sesta-MIBI ${ }^{\text {tc99. }}$.

Los resultados fueron expresados como media \pm DS, para lo cual se utilizó INFOSTAT 2008.

\section{Criterios de selección}

Ausencia de antecedentes o evidencia de enfermedad multiglandular (hiperparatiroidismo secundario, familiar o neoplasia endócrina múltiple), sospecha de carcinoma paratiroideo, hiperparatiroidismo persistente o recurrente, presencia de bocio, cirugía cervical previa y estudios de localización negativos o con localizaciones múltiples, siendo indispensable para realizar el abordaje mini-invasivo la coincidencia de la ecografía y el centellograma. Todos los pacientes firmaron un consentimiento informado para PTMIV y se les realizó una laringoscopía indirecta para determinar la función de las cuerdas vocales. Siempre se envió a biopsia por congelación la glándula resecada. No realizamos el mihpt.

Previo al alta se controló el calcio sérico, el cual fue nuevamente controlado a la semana, al mes, a los seis meses y al año de la cirugía. Controles posteriores se llevaron a cabo anualmente. EI dolor fue evaluado con una escala verbal del 0 al 10 a las 6 horas y previa al alta hospitalaria. El 0 representa ausencia de dolor y el 10 dolor muy intenso. Todos los pacientes fueron controlados ambulatoriamente a las cuatro semanas de la cirugía.

\section{Técnica quirúrgica}

La cirugía se lleva a cabo bajo anestesia general, con el paciente en posición supina y con el cuello levemente extendido. El cirujano se ubica a la derecha del paciente, el primer ayudante con la cámara frente al cirujano y el segundo a la cabeza del enfermo para presentar el campo quirúrgico.

La incisión de $15 \mathrm{~mm}$ se realiza a una distancia variable de la horquilla esternal en la línea media según la contextura de la persona y en particular a la conformación del cuello. Se disecan dos mínimos colgajos por debajo del músculo cutáneo y seccionamos la aponeurosis cervical superficial con electrobisturí cuidando siempre de no provocar lesiones térmicas en la piel y separamos los músculos infrahioideos de la glándula tiroides. Se crea el espacio operatorio mediante el uso de separadores pequeños, de extremo romo y atraumáticos llevando el lóbulo tiroideo hacia la línea media y los músculos del lado afectado hacia fuera con un segundo separador. Una óptica de $30^{\circ}$ y de $5 \mathrm{~mm}$ es introducida a través de la incisión. La disección de la corredera tiro-traqueal se lleva a cabo en forma exangüe con la ayuda de instrumental delicado. El primer vaso controlado, de estar presente, es la vena tiroidea media, luego la tiroides se separa de la tráquea y del esófago.

Después de identificar el nervio recurrente laríngeo del lado afectado, se procede a la disección de la glándula paratiroidea anormal previamente localizada. La magnificación de 
imágenes provista por la óptica facilita la identificación del nervio y de la glándula siempre y cuando la disección sea delicada y con hemostasia muy prolija. Localizada la glándula patológica se procede a la disección roma de su pedículo y su posterior sección con el empleo del bisturí harmónico. Extracción del adenoma, control estricto de hemostasia y colocación de surgicel en el lecho quirúrgico. Cierre de la línea media y del platisma con material reabsorbible. Piel con sutura intradérmica.

\section{Resultados}

En todos los pacientes la ubicación del adenoma paratiroideo señalado por los estudios de imágenes preoperatorios coincidió con los hallazgos quirúrgicos, los cuales fueron de localización superior en 7 casos (3 izquierdos) e inferiores en 20 casos ( 8 derechos), de los cuales, dos eran intra-tiroideos. En ambos casos después de una exploración cervical bilateral exhaustiva manteniendo el mismo abordaje y al encontrar las tres glándulas paratiroideas normales y no hallar la glándula patológica se decidió guiado por las imágenes, realizar una tiroidectomía derecha, encontrando los adenomas en el interior del lóbulo extraído. En uno de los casos tuvimos que ampliar la incisión en $10 \mathrm{~mm}$. Todos los pacientes presentaron normocalcemia en el postoperatorio y no hubo lesiones recurrenciales transitorias ni definitivas. No registramos ningún caso de persistencia del hiperparatiroidismo, definido como la presencia de hipercalcemia dentro de los 6 meses de la operación. El tiempo operatorio fue de $25 \pm 10$ minutos. En todos los tumores extraídos se confirmó el diagnóstico histológico de adenoma, con un peso promedio de 1,09 $\pm 1,05$ gramos. El dolor postoperatorio fue mínimo traducido en el escaso requerimiento de analgesia y en un promedio de 2,4 $\pm 1,7$ para la escala verbal del dolor. El promedio de estadía hospitalaria fue de $21,3 \pm 3,2$ horas.

\section{Discusión}

Actualmente la PTMIV es una opción válida en el tratamiento del HPT $1^{\circ}$, siendo considerada inclusive en varios centros hospitalarios como la primera opción terapéutica ${ }^{6-7}$. Autores como Duh $^{8}$ sostienen que la PTMIV ya no es un procedimiento en evaluación sino una técnica plenamente establecida, siendo el método de elección para el $92 \%$ de los grupos quirúrgicos de la Asociación Internacional de Cirujanos Endócrinos ${ }^{9-10}$.
Un criterio indispensable para decidir un abordaje mini-invasivo focalizado es tener dos estudios por imágenes de ubicación topográfica preoperatorios concordantes, que en nuestra experiencia y en la mayoría de los grupos de trabajo fue la ecografía cervical y el centellograma con Sesta-MIBI ${ }^{\text {tc99 }}$ 11-12-13-14-15. En nuestra serie la concordancia de ambos estudios predijo en forma satisfactoria la ubicación de todos lo adenomas, confirmando que la asociación ecografía-Sesta $\mathrm{MIBI}^{\mathrm{tc} 99}$ permite identificar el adenoma único en un porcentaje que va del 64 al 95\%, mostrando tasas más bajas cuando se usan por separado, del 71 al $80 \%$ para la ecografía y del 74 al $88 \%$ para el sesta-MIBI ${ }^{15-16}$. No objetivamos persistencias del HPT al igual que Udelsman ${ }^{11}$ en más de 600 paratiroidectomías mini-invasivas. Así mismo, con una media de seguimiento de 42,6 \pm 16,8 meses tampoco hemos observado recurrencias del HPT al igual Mihai ${ }^{17}$, ambos sin haber empleado el mipth.

Un aspecto fundamental en este tipo de cirugía es poder identificar con precisión la enfermedad multiglandular $^{12}$. Para tal fin, se utiliza actualmente junto con los estudios de localización preoperatoria el mipth debido a su ayuda para guiar la extensión del procedimiento sobre todo en el caso de imágenes discordantes, incrementando la eficacia de la cirugía, alcanzando un éxito quirúrgico del 97-99\% cuando las imágenes son coincidentes ${ }^{18}$.

Sin embargo, hay estudios que cuestionan la utilidad del mipth sobre todo en pacientes con estudios de localización coincidentes ${ }^{19-20}$, argumentando que el mipth pareciera solamente confirmar el hallazgo de las imágenes, como lo demostraran Delbridge $L$ y cols. ${ }^{15}$ en su serie con más de 1000 paratiroidectomías mini-invasivas y empleando el mipth, que el mismo no modificó la conducta quirúrgica y sólo confirmó los hallazgos pre-operatorios.

El tiempo operatorio de $25 \pm 10$ minutos fue similar al de otros grupos quirúrgicos una vez lograda la curva de aprendizaje, mostrando tiempos iniciales más prolongados ${ }^{17-21}$.

El escaso dolor creemos que está relacionado al tamaño mínimo de la incisión, a la menor disección, y a la menor extensión cervical requerida ${ }^{22}$. Todos los pacientes se mostraron muy satisfechos con el resultado estético de la incisión, resultado observado en otras publicaciones que emplean abordajes mínimos ${ }^{21}$. 22.

La estadía hospitalaria menor a un día, nos plantea la posibilidad de realizarla en un futuro en el marco de la cirugía ambulatoria. 


\section{Conclusión}

En base a nuestros resultados y a la literatura consultada, creemos que el abordaje miniinvasivo con magnificación de imágenes, con exploración unilateral de las paratiroides sin mipth, bajo la guía de 2 estudios de localización concordantes es un procedimiento seguro y factible, con buenos resultados estéticos, escaso dolor post operatorio y estancia hospitalaria reducida.

\section{Bibliografía}

1.- Tibblin S, Bodenson AG, Ljungberg O. Unilateral parathyroidectomy in hyperparathyroidism due to single adenoma. Ann Surg 1982, 195: 245-252.

2.- Gagner M. Endoscopic subtotal parathyroidectomy in patients with primary hyperparathyroidism. Br J Surg 1996; 83: 875.

3.- Miccoli P, Materazzi G. Cirugía cervical endoscópica. CirEsp 2005; 77: 181-186.

4.- Henry JF, Defechereux T, Gramática L, et al. Minimally invasive videoscopicparathyroidectomy by lateral approach.Langenbecks Arch Surg 1999; 384: 298-301.

5.- Ikeda Y, Takami $H$, Sasaki Y, et al. Endoscopic neck surgery by the axilary approach. $\mathrm{J}$ am CollSurh 2000; 191: 336-340.

6.- Henry JF, Jacobone M, Mirallie M, et al. Indications and results of video assisted parathyroidectomy by a lateral approach in patients with a primary hyperparathyroidism. Surgery 2001; 130: 999-1004.

7.- Berti $P$, Materazzi G, Miccoli $P$, et al. Limits and drawbacks of video-assisted parathyroidectomy. $\mathrm{Br} \mathrm{J}$ Surg 2003; 90: 743-747.

8.- Duh QY. Presidential address: minimally invasive endocrine surgery -Standard of treatment or hype? Surgery 2003;134:849-57.

9.- Agarwal G, Barraclough BH, Robinson BG, et al. Minimally invasive parathyroidectomy using the 'focused' lateral approach. Results of the first 100 consecutive cases.ANZ J Surg2002; 72: 100-104.

10.- Bergenfelz A, Lindblom P, Tibblin S, et al. Unilateral versusbilateral neck exploration for primary hyperparathyroidism: $A$ prospective randomized controlled trial. Ann Surg 2002; 236: 543-551.

11.- Udelsman R. Six hundred fifty-six consecutive explorations for primary hyperparathyroidism. Ann Surg 2002; 5:665-672.

12.- Guerrero M, Clark O. A comprehensive review of intraoperative parathyroid hormone monitoring. WJOES 2010; 2: 21-27.

13.- Henry JF, Misso C, Sebag F, et al. laparatiroidectomia mini-invasiva video-assistita con approcciolateraleneipazienti con iperparatiroidismo primario. Ann ItalChir 2003; 74: 401-405.

14.- Lombardi CP, Raffaelli M, Traini E, et al. Advantages of a video-assisted approach to parathyroidectomy. ORL J OtorhinolaryngolRelat Spec 2008; 70: 313-318.

15.- Suliburk JW, Sywak MS, Delbridge LW et al. 1000 minimally invasive parathyroidectomies without intraoperative parathyroid hormone measurement: lessons learned. ANZ J Surg 2011; 81: 362-365.

16.- Gawande $A A$, et al. Reassessment of parathyroid hormone monitoring during parathyroidectomy for primary hyperparathyroidism after 2 preoperative localization studies. Arch Surg 2006; 141: 381-384.
17.- Mihai R, Palazzo F, Gleeson F, Sadler G. Minimally invasive parathyroidectomy without intraoperative parathyroid hormone monitoring in patiens with primary hyperparathyroidism. Br J Surg 2007, 94: 42-47.

18.- Perrier ND, et al. Intraoperative parathyroid aspiration and parathyroid hormone assay as an alternative to frozen section for tissue identification. World $J$ Surg 2000; 24: 1319-1322.

19.- Chen $\mathrm{H}$, et al. Intraoperative parathyroid hormone testing improves cure rates in patients undergoing minimally invasive parathyroidectomy. Surgery 2005; 138: 583-587. 6.

20.- Stalberg $P$, et al. Intraoperative parathyroid hormone measurement during minimally invasive parathyroidectomy: Does it "value-add" to decisionmaking? J Am CollSurg 2006; 203: 1 - 6.

21.- Miccoli P, Berti P, Materazzi G, et al. Results of videoassisted parathyroidectomy: single institution's six year experience. World J Surg 2004, 28: 1216-1218.

22.- Miccoli $P$, Berti $P$, Materazzi $G$, et al. Minimally invasive video-assisted thyroidectomy: Five years of experience. J Am CollSurg 2004; 199: 243-248. 